\title{
SYNTAKTISCHE EXPONENTEN UND ZAHLMARKER DER UNREGELMÄSSIGEN VERBEN IM PRÄSENS INDIKATIV
}

Im Folgenden werden Analysen der verbalen unregelmäßigen Formen ${ }^{1}$ des Gegenwartsdeutschen vorgenommen. Die theoretischen Grundlagen sind Darski's linguistischem Analysemodell (2004) sowie seiner neuesten Grammatik (2010) entnommen worden. Mit dem Analysemodell wird ein tragfähiges Analyseinstrumentarium dargeboten, welches u.a. erlaubt, alle flektierenden Wortformen hinsichtlich der von ihnen ausgedrückten grammatischen Kategorien bzw. Funktionen formimmanent und einheitlich zu beschreiben. Die Methode geht auf die Erkenntnis zurück, dass die bisherige, in der wissenschaftlichen Beschreibung fast allgegenwärtige, Verwendung der Begriffe „Flexionsendung“, „Stamm“, „Wurzel“ und „Suffix“ von Widersprüchen belastet und vor allem auf keine klare Theorie bzw. Definition zurückführbar ist ${ }^{2}$. Der methodologische Missstand kann nur behoben werden, indem man objektive, eindeutig abgrenzbare Analysekriterien einsetzt. Laut Darski sind die zu untersuchenden Wortformen in Singular- bzw. Pluralstämme und syntaktische Singular- und Pluralexponenten zu zerlegen. Weitere, auf den Vergleich der Singular- und Pluralstämme beruhende Analysen ermöglichen die Festlegung der neutralen Flexionsstämme sowie Zahlmarker (Singular- und Pluralmarker). Die vorstehende Analyse zielt darauf ab, dies widerspruchsfrei durchzuführen. Auf dieser Grundlage lassen sich neuartige Konjugationsparadigmen und regeln erarbeiten, zumal die in der einschlägigen Literatur vorgeschlagenen Konjugationsmuster in der Regel nicht alle (manchmal sogar nur einige wenige) Verben umfassen, sodass das Konjugationssystem nicht vollständig präsentiert wird, was zweifelsohne dem Deutsch Lernenden Schwierigkeiten bereitet (Vgl. u.a.: Jung 1955: 227-231; Weinrich 1993: 183-198; Eisenberg 1989: 110-115; Helbig, Buscha 2001: 26-40; Engel 2004: 216-223; DUDEN. Die Grammatik 2005: 483-491).

\footnotetext{
${ }^{1}$ Zur Definition der regelmäßigen und unregelmäßigen Verben vgl. Darski 2010: $234 f$.

${ }^{2}$ Zur Kritik vgl. u.a. Darski 2010: 122ff.
} 
Den Ausgangspunkt für die Wortanalyse bilden die Darskischen primären, sekundären und tertiären Wortformen (Vgl. Darski 2010: 105-121). Vergleicht man Wortformen einer Sprache miteinander, so stellt man fest, dass eine Gruppe der Wortformen solche bilden, die sich in ihrer Bedeutung nur dadurch unterscheiden, ob das durch sie Genannte einmal oder wenigstens zweimal vorhanden ist, d.h. im Singular oder im Plural steht. Der Unterschied zwischen den Wortformen im Singular und Plural kommt meistens durch die Änderung ihrer Form zum Ausdruck (Vgl. Darski 2010: 125). In diesem Zusammenhang spricht Darski von den sog. Singularund Plurallexemen ${ }^{3}$.

Die Wortformen der Singularlexeme werden zum Vergleich in Konjugationsparadigmen ${ }^{4}$ orthographisch und phonologisch zusammengestellt, z.B. die Singularlexeme der Verben kennen, backen und sein:

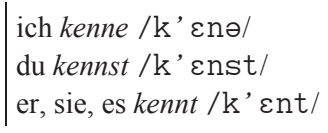

Die sprachlichen Mittel, die sich in allen Wortformen eines Singularlexems wiederholen, die im Falle eines regelmäßigen Wechsels durch einen bzw. mehrere große Buchstaben neutral dargestellt werden, die also dieselbe Bedeutung aller Wortformen eines Singularlexems sichern, bilden den (neutralen) Singularstamm.

Die sprachlichen Mittel, die dieselbe Bedeutung eines Singularlexems nicht sichern, die aber in den Realisierungen der Wortformen desselben Singularlexems vorhanden sind, werden als syntaktische Singularexponenten betrachtet.

(Darski 2010: 129)

Der Definition des neutralen Singularstamms zufolge sind als neutrale Singularstämme:

- kenn /k’ $\mathrm{n}$ / vom Verb kennen,

- $b \ddot{A} c k / \mathrm{b}$ ' yk/ vom Verb backen

zu betrachten. Zu den syntaktischen Singularexponenten gehören:

\begin{tabular}{|c|c|}
\hline $\begin{array}{l}\text { kenn /k’ } \mathrm{kn} / \\
\text { - } e / \text { / / für die 1. Person Singular Präsens } \\
\text { Indikativ } \\
\text { - } s t / \text { st/ für die 2. Person Singular Präsens } \\
\text { Indikativ } \\
\text { - } t / t / \text { für die 3. Person Singular Präsens } \\
\text { Indikativ }\end{array}$ & 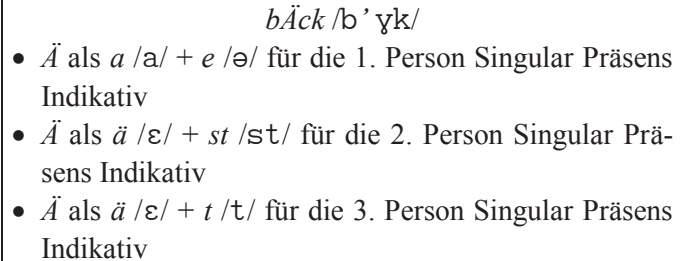 \\
\hline
\end{tabular}

${ }^{3}$ Zur Definitionen der Singular- und Plurallexeme vgl. Darski 2010: 125.

${ }^{4}$ Darski gebraucht den Begriff „Paradigma“ im Sinne von Engel (1977: 877). Vgl. Darski 2010: 126. 
Den Wechsel $a / \mathrm{a} / z u \ddot{a} / \varepsilon /$ kann man auch bei anderen Verben feststellen, z.B. bei fangen, waschen. Aus diesem Grund wird der Buchstaben- bzw. Phonemwechsel als regelmäßig angesehen (Vgl. Darski 2010: 127).

Beim Verb sein lässt sich aber nicht feststellen, was als neutraler Singularstamm und was als syntaktische Singularexponenten zu gelten haben. Da der Wechsel in diesem Fall unregelmäßig ist, ist die ganze Wortform zugleich Singularstamm und syntaktischer Singularexponent.

Nun werden die Wortformen der Plurallexeme zum Vergleich in Konjugationsparadigmen zusammengestellt und analysiert wie die Singularlexeme, z.B. die Plurallexeme der Verben kennen, backen und sein:

wir kennen /k' ' nən/
ihr kennt /k' ' nt/
sie, Sie kennen /k’ Enən/

wir backen /b' aky/

ihr backt/b' akt/

sie, Sie backen /b’akn/

wir sind / z' Int/
ihr seid /z' a ع t/
sie, Sie sind /z' Int/

Die sprachlichen Mittel, die sich in allen Wortformen eines Plurallexems wiederholen, die im Falle eines regelmäßigen Wechsels durch einen bzw. mehrere große Buchstaben neutral dargestellt werden, die also dieselbe Bedeutung aller Wortformen eines Plurallexems sichern, bilden den (neutralen) Pluralstamm.

Die sprachlichen Mittel, die dieselbe Bedeutung eines Plurallexems nicht sichern, die aber in den Realisierungen der Wortformen desselben Plurallexems vorhanden sind, werden als syntaktische Pluralexponenten betrachtet.

(Darski 2010: 129)

Der Definition des neutralen Pluralstamms zufolge sind als neutrale Pluralstämme:

- kenn /k’ $\varepsilon \mathrm{n} /$ vom Verb kennen,

- back/b' ak/ vom Verb backen

zu betrachten. Zu den syntaktischen Pluralexponenten gehören:

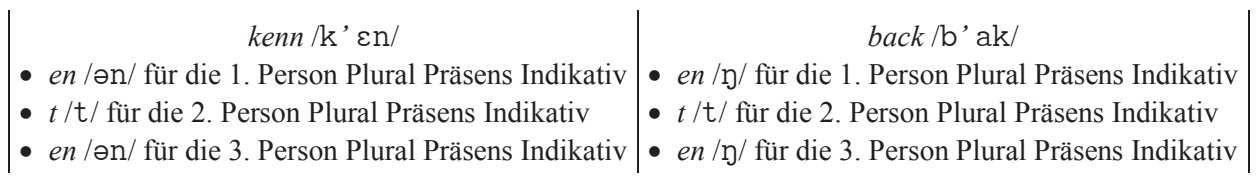

Beim Verb sein lässt sich wiederum nicht feststellen, was als neutraler Pluralstamm und was als syntaktische Pluralexponenten zu gelten haben. Der Wechsel ist auch in diesem Fall unregelmäßig, deswegen ist die ganze Wortform zugleich Pluralstamm und syntaktischer Pluralexponent. 
Nachdem die Definitionen des Singular- und Pluralstamms sowie der syntaktischen Singular- und Pluralexponenten erläutert worden sind, werden die Definitionen des neutralen Flexionstamms sowie der Singular- und Pluralmarker präsentiert.

Die sprachlichen Mittel, die sich sowohl in dem (neutralen) Singularstamm als auch in dem ihm in der Bedeutung entsprechenden (neutralen) Pluralstamm wiederholen beziehungsweise einen regelmäßigen Wechsel aufweisen, der durch einen oder mehrere große Buchstaben neutral dargestellt wird, bilden den in der Zahl nicht markierten Stamm, der der Einfachheit halber kurz (neutraler) Flexionsstamm genannt wird.

(Darski 2010: 132)

Zur Veranschaulichung der Definition werden zwei Beispiele angeführt: die Verben kennen und backen.

\begin{tabular}{|c|c|c|c|c|c|}
\hline Singularstamm & Pluralstamm & Flexionsstamm & Singularstamm & Pluralstamm & Flexionsstamm \\
\hline kenn/k' $\varepsilon \mathrm{n} /$ & kenn /k'sn/ & kenn /k' $\varepsilon \mathrm{n} /$ & b̈̈ck /b' yk/ & back/b'ak/ & bAck /b'ak/ \\
\hline
\end{tabular}

An dieser Stelle sind die Definitionen der Zahlmarker (Singular- und Pluralmarker) einzuführen.

Das sprachliche Mittel/Die sprachlichen Mittel, durch das/die sich der (neutrale) Singular- und Pluralstamm vom neutralen Flexionsstamm unterscheidet/unterscheiden, wird/werden als Zahlmarker bezeichnet.

Das sprachliche Mittel/Die sprachlichen Mittel, durch das/die sich der (neutrale) Singularstamm vom neutralen Flexionsstamm unterscheidet/unterscheiden, wird/werden als Singularmarker bezeichnet.

Das sprachliche Mittel/Die sprachlichen Mittel, durch das/die sich der (neutrale) Pluralstamm vom neutralen Flexionsstamm unterscheidet/unterscheiden, wird/werden als Pluralmarker bezeichnet.

(Darski 2010: 132f.)

Als Singularmarker des Verbs backen gelten:

- $A$ als $\ddot{a} / \varepsilon /$

- e/al

Im Falle dieses Verbs sind der Flexionsstamm und der Singularstamm nicht formengleich, sodass syntaktische Singularexponenten monofunktional sind ${ }^{5}$. Wenn aber der Flexionsstamm und der Singular- bzw. Pluralstamm formengleich sind, dann kennzeichnen die Zahl die syntaktischen Singular- bzw. Pluralexponenten. Als Singularmarker des Verbs kennen gelten seine syntaktischen Singularexponenten:

${ }^{5}$ Vgl. Darski 2010: 133. Monofunktional können aber auch syntaktische Pluralexponenten sein. 
- $e / \partial /$

- $s t / \mathrm{st} /$

- $t / \mathrm{t} /$

Dasselbe betrifft die Pluralmarker der Verben kennen und backen: Ihre syntaktischen Pluralexponenten sind zugleich Pluralmarker:

\begin{tabular}{|c|c|}
\hline $\begin{array}{ll} & \text { kennen } \\
\text { - } & \text { en /ən/ } \\
\text { - } t / \mathrm{t} / & \\
\text { - } & \text { en /ən/ }\end{array}$ & $\begin{array}{l}\text { - } e n / \mathrm{y} / \\
\text { - } t / \mathrm{t} / \\
\text { - } e n / \mathrm{y} /\end{array}$ \\
\hline
\end{tabular}

In solchen Fällen sind die syntaktischen Singular- bzw. Pluralexponenten bifunktional, d.h. sie zeigen die syntaktische Funktion sowie die Zahl an (Vgl. Darski 2010: 133).

Als Gegenstand der Analyse gelten unregelmäßige Verben im Präsens Indikativ. Unberücksichtigt bleiben dabei die Präfigierungen, z.B. anbieten, verbieten etc., weil sie dieselben Formen wie einfache Verben aufweisen. Präfigierte Verben werden nur dann analysiert, wenn es die entsprechenden einfachen Verben nicht gibt, z.B. verlieren $v \mathrm{~s}$. *lieren oder zu den regelmäßigen Verben gezählt werden, z.B. befehlen $v$ s. fehlen. Insgesamt werden 193 Verben analysiert. Dabei ist aber zu betonen, dass einige mehr Konjugationsmöglichkeiten im Singular und/oder im Plural haben, was die Zahl der syntaktischen Exponenten, Zahlmarker sowie Singular-, Plural- und Flexionsstämme beeinflusst, z.B.: das Verb fechten.
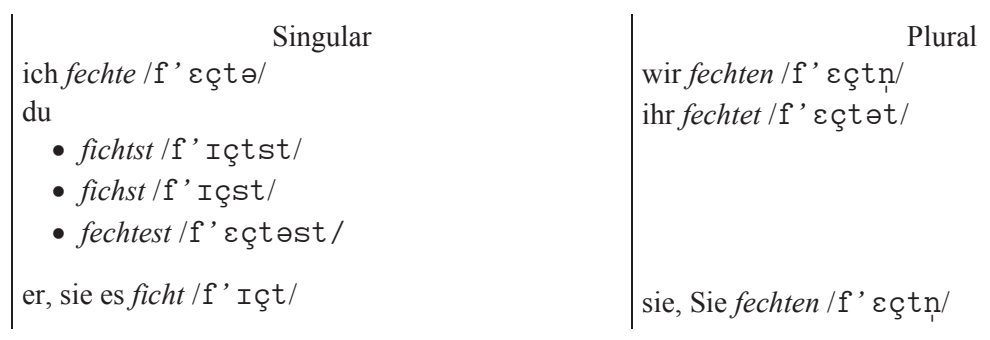

Es gibt aber auch Verben, die nur in der 3. Person Singular konjugiert vorkommen, z.B. dünken (mir/mich dünkt/d'y ykt/ bzw. deucht/d' oœeçt/), was andererseits die Zahl der Pluralstämme, der syntaktischen Pluralexponenten und der Pluralmarker eingrenzt.

$\mathrm{Zu}$ bemerken sei noch, dass die Analysen auf der phonologischen und orthographischen Ebene verlaufen. Die phonologischen sprachlichen Mittel werden mithilfe der Zeichen des „Internationalen Phonetischen Alphabets“ wiedergegeben. Es wird hier von der hohen bis mittleren Artikulationspräzision ausgegangen. Bei den Transkriptionen richte ich mich nach dem „Deutschen Aussprachewörterbuch“ von Eva- 
Maria Krech, Eberhard Stock, Ursula Hischfeld und Lutz Christian Anders (2009). $\mathrm{Zu}$ den orthographischen sprachlichen Mittel gehören dagegen die Buchstaben und ihre Anordnung. Die auf zwei Ebenen durchgeführten Analysen haben aber zur Folge, dass die Zahl der ermittelten syntaktischen Exponenten und Zahlmarker größer ist, als im Falle der eindimensionalen (orthographischen bzw. phonologischen) Analysen. Dabei wird demonstriert, inwieweit die Erkenntnisse einer phonologisch orientierten Analyse von denen einer abweichen, deren Gegenstand die orthographischen Einheiten sind. Das beweist, dass eine eindeutige Spezifizierung des Untersuchungsgegenstandes einer grammatischen Klassifizierung notwendig ist, wobei diese den traditionellen Modellen nur selten zugrunde liegt.

Die ermittelten syntaktischen Exponenten sowie Zahlmarker werden in Tabellen (geordnet nach der Häufigkeit ihrer Realisierung) zusammengestellt.

Beim ersten Anlauf werden syntaktische Singularexponenten besprochen und zusammengestellt. Im zweiten Teil wende ich mich den syntaktischen Pluralexponenten zu, um durch den Vergleich der Singular- mit Pluralstämmen zu neutralen Flexionsstämmen zu gelangen. Schließlich werden Singular- und Pluralmarker festund zusammengestellt.

Die Analyse hat 42 Gruppen der syntaktischen Singularexponenten ergeben, was die folgende Tabelle veranschaulicht.

Tabelle Nr. 1: Syntaktische Singularexponenten für das Präsens Indikativ

\begin{tabular}{|c|c|c|c|}
\hline & Syntaktische Singularexponenten & $\begin{array}{l}\text { Zahl der Verben, die die } \\
\text { syntaktischen Singula- } \\
\text { rexponenten aufweisen }\end{array}$ & $\begin{array}{c}\text { Beispiel mit } \\
\text { Singularstamm }\end{array}$ \\
\hline 1. & $\begin{array}{l}\text { 1.P.Sg.: e /ə/ } \\
\text { 2.P.Sg.: st /st/ } \\
\text { 3.P.Sg.: } \mathrm{t} / \mathrm{t} /\end{array}$ & 61 & $\begin{array}{l}\text { beginnen } \\
\text { beginn /bəg' In/ }\end{array}$ \\
\hline 2. & $\begin{array}{l}\text { 1.P.Sg.: } \varnothing^{6} \\
\text { 2.P.Sg.: st /st/ } \\
\text { 3.P.Sg.: } \mathrm{t} / \mathrm{t} /\end{array}$ & 19 & $\begin{array}{l}\text { leiden } \\
\text { leide /1' aદ્də/ }\end{array}$ \\
\hline 3. & $\begin{array}{l}\text { 1.P.Sg.: e /ə/ } \\
\text { 2.P.Sg.: t /t/ } \\
\text { 3.P.Sg.: t /t/ }\end{array}$ & 17 & 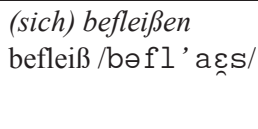 \\
\hline 4. & $\begin{array}{l}\text { 1.P.Sg.: } K \text { als } g / g /+\mathrm{e} / ə / \\
\text { 2.P.Sg.: } K \text { als g } / \mathrm{k} /+\mathrm{st} / \mathrm{st} / \\
\text { 3.P.Sg.: } \mathrm{K} \text { als } \mathrm{g} / \mathrm{k} /+\mathrm{t} / \mathrm{t} /\end{array}$ & 11 & $\begin{array}{l}\text { biegen } \\
\text { bieg /b’ i : H/ }\end{array}$ \\
\hline 5. & $\begin{array}{l}\text { 1P.Sg.: I als e /E/ + e /ə/ } \\
\text { 2.P.Sg.: I als } \mathrm{i} / \mathrm{I} /+\mathrm{st} / \mathrm{st} / \\
\text { 3.P.Sg.: I als } \mathrm{i} / \mathrm{I} /+\mathrm{t} / \mathrm{t} /\end{array}$ & 11 & $\begin{array}{l}\text { dreschen } \\
\text { drIsch /db' I S/ }\end{array}$ \\
\hline
\end{tabular}

${ }^{6}$ Das Symbol wird jeweils verwendet zur Bezeichnung fehlender syntaktischer Exponenten. 
Syntaktische Exponenten und Zahlmarker der unregelmässigen Verben im Präsens Indikativ 107

\begin{tabular}{|c|c|c|c|}
\hline & Syntaktische Singularexponenten & $\begin{array}{l}\text { Zahl der Verben, die die } \\
\text { syntaktischen Singula- } \\
\text { rexponenten aufweisen }\end{array}$ & $\begin{array}{c}\text { Beispiel mit } \\
\text { Singularstamm }\end{array}$ \\
\hline 6. & $\begin{array}{l}\text { 1.P.Sg.: } \mathrm{P} \text { als } \mathrm{b} / \mathrm{b} /+\mathrm{e} / ə / \\
\text { 2.P.Sg.: } \mathrm{P} \text { als } \mathrm{b} / \mathrm{p} /+\mathrm{st} / \mathrm{st} / \\
\text { 3.P/Sg.: } \mathrm{P} \text { als } \mathrm{b} / \mathrm{p} /+\mathrm{t} / \mathrm{t} /\end{array}$ & 9 & $\begin{array}{l}\text { heben } \\
\text { heP /h' e : } \ominus /\end{array}$ \\
\hline 7. & $\begin{array}{l}\text { 1.P.Sg.: } \mathrm{R} \text { als } \mathrm{r} / \mathrm{B} /+\mathrm{e} / \ominus / \\
\text { 2.P.Sg.: } \mathrm{R} \text { als } \mathrm{r} / \mathrm{e} /+\mathrm{st} / \mathrm{st} / \\
\text { 3.P.Sg.: } \mathrm{R} \text { als } \mathrm{r} / \mathrm{e} /+\mathrm{t} / \mathrm{t} /\end{array}$ & 8 & 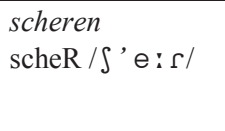 \\
\hline 8. & $\begin{array}{l}\text { 1.P.Sg.: } \varnothing \\
\text { 2.P.Sg.: st /st/ } \\
\text { 3.P.Sg.: } \varnothing\end{array}$ & 6 & $\begin{array}{l}\text { können } \\
\text { kann /k' an/ }\end{array}$ \\
\hline 9. & $\begin{array}{l}\text { 1.P.Sg.: I als e } / \varepsilon /+\mathrm{e} / \curvearrowright / \\
\text { 2.P.Sg.: I als } \mathrm{i} / \mathrm{I} / \mathrm{t} / \mathrm{t} / \\
\text { 3.P.Sg.: I als } \mathrm{i} / \mathrm{I} /+\mathrm{t} / \mathrm{t} /\end{array}$ & 5 & $\begin{array}{l}\text { essen } \\
\text { Iss /?' IS/ }\end{array}$ \\
\hline 10. & $\begin{array}{l}\text { 1.P.Sg.: } \mathrm{Ä} \text { als a } / \mathrm{a} /+\mathrm{e} / ə / \\
\text { 2.P.Sg.: } \mathrm{A} \text { als ä } / \varepsilon /+\mathrm{st} / \mathrm{st} / \\
\text { 3.P.Sg.: } \mathrm{Ä} \text { als ä } / \varepsilon /+\mathrm{t} / \mathrm{t} /\end{array}$ & 4 & $\begin{array}{l}\text { fallen } \\
\text { fÄll /f' ' } \mathrm{l} \text { l/ }\end{array}$ \\
\hline 11. & $\begin{array}{l}\text { 1.P.Sg.: IEH als eh /e : / e /ə/ } \\
\text { 2.P.Sg.: IEH als ieh } / \mathrm{i}: /+\mathrm{st} / \text { st/ } \\
\text { 3.P.Sg.: IEH als ieh } / \mathrm{i}: /+\mathrm{t} / \mathrm{t} /\end{array}$ & 4 & 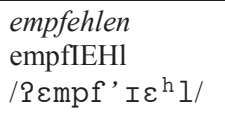 \\
\hline 12. & $\begin{array}{l}\text { 1.P.Sg.: } \mathrm{S} \text { als } \mathrm{s} / \mathrm{z} /+\mathrm{e} / \mathrm{\theta} / \\
\text { 2.P.Sg.: } \mathrm{S} \text { als } \mathrm{s} / \mathrm{s} /+\mathrm{t} / \mathrm{t} / \\
\text { 3.P.Sg.: } \mathrm{S} \text { als } \mathrm{s} / \mathrm{s} /+\mathrm{t} / \mathrm{t} /\end{array}$ & 4 & 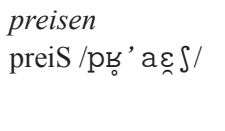 \\
\hline 13. & $\begin{array}{l}\text { 1.P.Sg.: I als e } / \mathcal{E} /+\mathrm{e} / \partial / \\
\text { 2.P.Sg.: I als } \mathrm{i} / \mathrm{I} /+\mathrm{st} / \mathrm{st} / \\
\text { 3.P.Sg.: I als } \mathrm{I} / \mathrm{I} /\end{array}$ & 4 & $\begin{array}{l}\text { gelten } \\
\text { gllt/g' Ilt/ }\end{array}$ \\
\hline 14. & $\begin{array}{l}\text { 1.P.Sg.: I als e } / \varepsilon /+P \text { als } b / b /+\mathrm{e} / \oslash / \\
\text { 2.P.Sg.: I als } \mathrm{i} / \mathrm{I} /+\mathrm{P} \text { als } \mathrm{b} / \mathrm{p} /+\mathrm{st} / \mathrm{st} / \\
\text { 3.P.Sg.: I als } \mathrm{i} / \mathrm{I} /+\mathrm{P} \text { als } \mathrm{b} / \mathrm{p} /+\mathrm{t} / \mathrm{t} /\end{array}$ & 3 & $\begin{array}{l}\text { sterben } \\
\operatorname{stIrP} / \int t^{\prime} I^{\mathrm{B}} \theta /\end{array}$ \\
\hline 15. & $\begin{array}{l}\text { 1.P.Sg.: } \ddot{\mathrm{A}} \text { als a } / \mathrm{a}: /+\mathrm{e} / ə / \\
\text { 2.P.Sg.: } \mathrm{A} \text { als ä } / \varepsilon: /+\mathrm{st} / \mathrm{st} / \\
\text { 3.P.Sg.: } \mathrm{A} \text { als ä } / \varepsilon: /\end{array}$ & 2 & $\begin{array}{l}\text { raten } \\
\text { rÄt/b'yt/ }\end{array}$ \\
\hline 16. & $\begin{array}{l}\text { 1.P.Sg.: I als e } / \varepsilon /+\mathrm{t} / \mathrm{t} /+\mathrm{e} / \curvearrowright / \\
\text { 2.P.Sg.:I als } \mathrm{i} / \mathrm{I} /+\mathrm{st} / \mathrm{st} / \\
\text { 3.P.Sg.: I als } \mathrm{i} / \mathrm{I} /+\mathrm{t} / \mathrm{t} /\end{array}$ & 2 & $\begin{array}{l}\text { fechten } \\
\text { fIch /f' IÇ/ }\end{array}$ \\
\hline 17. & $\begin{array}{l}\text { 1.P.Sg.: } \mathrm{E} \text { als e } / \varepsilon /+\mathrm{e} / \partial / \\
\text { 2.P.Sg.: } \mathrm{E} \text { als e } / \varepsilon /+ \text { est } / \text { əst } / \\
\text { 3.P.Sg.: } \mathrm{E} \text { als } \mathrm{i} / \mathrm{I} /\end{array}$ & 2 & $\begin{array}{l}\text { flechten } \\
\text { flEcht /f I’ } \varepsilon c ̧ t /\end{array}$ \\
\hline 18. & $\begin{array}{l}\text { 1.P.Sg.: } \ddot{A} \text { als a } / \mathrm{a} /+\mathrm{e} / \ominus / \\
\text { 2.P.Sg.: } \ddot{\mathrm{A}} \text { als } \mathrm{a} / \varepsilon /+\mathrm{t} / \mathrm{t} / \\
\text { 3.P.Sg.: } \ddot{\mathrm{A}} \text { als } \ddot{a} / \varepsilon /+\mathrm{t} / \mathrm{t} /\end{array}$ & 2 & $\begin{array}{l}\text { lassen } \\
1 \text { Ï̈ss /1 ’ ys/ }\end{array}$ \\
\hline
\end{tabular}




\begin{tabular}{|c|c|c|c|}
\hline & Syntaktische Singularexponenten & $\begin{array}{l}\text { Zahl der Verben, die die } \\
\text { syntaktischen Singula- } \\
\text { rexponenten aufweisen }\end{array}$ & $\begin{array}{c}\text { Beispiel mit } \\
\text { Singularstamm }\end{array}$ \\
\hline 19. & $\begin{array}{l}\text { 1.P.Sg.: ÄU als au /ao/ + e /ə/ } \\
\text { 2.P.Sg.: ÄU als äu /oœ/ + st /st/ } \\
\text { 3.P.Sg.: ÄU als äu /oœ/ + t/t/ }\end{array}$ & 2 & $\begin{array}{l}\text { laufen } \\
\text { läUf / ’ yuf/ }\end{array}$ \\
\hline 20. & $\begin{array}{l}\text { 1.P.Sg.: } \ddot{A} \text { als a } / \mathrm{a}: /+\mathrm{K} \text { als g } / \mathrm{g} /+\mathrm{e} / \oslash / \\
\text { 2.P.Sg.: Ä als ä } / \varepsilon: /+\mathrm{K} \text { als g } / \mathrm{k} /+\mathrm{st} / \mathrm{st} / \\
\text { 3.P.Sg.: } \mathrm{A} \text { als ä } / \varepsilon: /+\mathrm{K} \text { als g } / \mathrm{k} /+\mathrm{t} / \mathrm{t} /\end{array}$ & 2 & $\begin{array}{l}\text { schlagen } \\
\text { schlÄK / I I УН/ }\end{array}$ \\
\hline 21. & $\begin{array}{l}\text { 1.P.Sg.: I als e } / \varepsilon /+\mathrm{K} \text { als g } / \mathrm{g} /+\mathrm{e} / \ni / \\
\text { 2.P.Sg.: I als } \mathrm{i} / \mathrm{I} /+\mathrm{K} \text { als } \mathrm{g} / \mathrm{k} /+\mathrm{st} / \mathrm{st} / \\
\text { 3.P.Sg.: I als } \mathrm{i} / \mathrm{I} /+\mathrm{K} \text { als g } / \mathrm{k} /+\mathrm{t} / \mathrm{t} /\end{array}$ & 1 & $\begin{array}{l}\text { bergen } \\
\mathrm{b} \operatorname{IrK} / \mathrm{b}^{\prime} \mathrm{I}^{\mathrm{B}} \mathrm{H} /\end{array}$ \\
\hline 22. & $\begin{array}{l}\text { 1.P.Sg.: I als e } / \mathrm{e}: /+\mathrm{P} \text { als b } / \mathrm{b} /+\mathrm{e} / \mathrm{\vartheta} / \\
\text { 2.P.Sg.: I als } \mathrm{i} / \mathrm{i}: /+\mathrm{P} \text { als b } / \mathrm{p} /+\mathrm{st} / \mathrm{st} / \\
\text { 2.P.Sg.: I als } \mathrm{i} / \mathrm{i}: /+\mathrm{P} \text { als } \mathrm{b} / \mathrm{p} /+\mathrm{t} / \mathrm{t} /\end{array}$ & 1 & $\begin{array}{l}\text { geben } \\
\text { gIP/g' I } /\end{array}$ \\
\hline 23. & $\begin{array}{l}\text { 1.P.Sg.: I als e } / \mathcal{E} /+\mathrm{e} / \partial / \\
\text { 2.P.Sg.: I als } \mathrm{i} / \mathrm{I} / \\
\text { 3.P.Sg.: I als } \mathrm{i} / \mathrm{I} /\end{array}$ & 1 & $\begin{array}{l}\text { bersten } \\
\text { bIrst /b’ I }{ }^{\mathrm{B}} \mathrm{st} /\end{array}$ \\
\hline 24. & $\begin{array}{l}\text { 1.P.Sg.: I als e } / \mathrm{e}: /+\mathrm{e} / \partial / \\
\text { 2.P.Sg.: I als } \mathrm{i} / \mathrm{I} /+\mathrm{st} / \mathrm{st} / \\
\text { 3.P.Sg.: } \mathrm{I} \text { als } \mathrm{i} / \mathrm{I} /+\mathrm{t} / \mathrm{n} /\end{array}$ & 1 & $\begin{array}{l}\text { treten } \\
\text { trIt /t } \mathrm{t}_{\mathrm{B}} \text { 。It/ }\end{array}$ \\
\hline 25. & $\begin{array}{l}\text { 1.P.Sg.: I als e } / \varepsilon /+ \text { de } / \text { də/ } \\
\text { 2.P.Sg.: I als i } / \text { I }+ \text { st } / \text { st/ } \\
\text { 3.P.Sg.: I als i } / I /+d / t /\end{array}$ & 1 & $\begin{array}{l}\text { werden } \\
\mathrm{WIr} / \mathrm{V}^{\prime} \mathrm{I}^{\mathrm{B}} /\end{array}$ \\
\hline 26. & $\begin{array}{l}\text { 1.P.Sg.: IE als ä } / \varepsilon: /+\mathrm{R} \text { als } \mathrm{r} / \mathrm{b} /+\mathrm{e} / \oslash / \\
\text { 2.P.Sg.: IE als ie } / \mathrm{i}: /+\mathrm{R} \text { als } \mathrm{r} / /+\mathrm{st} / \mathrm{st} / \\
\text { 3.P.Sg.: IE als ie } / i: /+\mathrm{R} \text { als } \mathrm{r} / / /+\mathrm{t} / \mathrm{t} /\end{array}$ & 1 & $\begin{array}{l}\text { gebären } \\
\text { gebIER /gəb' Iع / / }\end{array}$ \\
\hline 27. & $\begin{array}{l}\text { 1.P.Sg.: IE als e } / \mathrm{e}: /+\mathrm{S} \text { als } \mathrm{s} / \mathrm{z} /+\mathrm{e} / \text { / } / \\
\text { 2.P.Sg.: IE als ie } / \mathrm{i}: /+\mathrm{S} \text { als } \mathrm{s} / \mathrm{s} /+\mathrm{t} / \mathrm{t} / \\
\text { 3.P.Sg.: IE als ie } / \mathrm{i}: /+\mathrm{S} \text { als } \mathrm{s} / \mathrm{s} /+\mathrm{t} / \mathrm{t} /\end{array}$ & 1 & $\begin{array}{l}\text { lesen } \\
\text { lIES /1’ IE / }\end{array}$ \\
\hline 28. & $\begin{array}{l}\text { 1.P.Sg.: I als } \ddot{\mathrm{o}} / \circledast /+\mathrm{e} / \oslash / \\
\text { 2.P.Sg.: I als } \mathrm{i} / \mathrm{I} /+\mathrm{st} / \mathrm{st} / \\
\text { 3.P.Sg.: } \mathrm{I} \text { als } \mathrm{i} / \mathrm{I} /+\mathrm{t} / \mathrm{t} /\end{array}$ & 1 & $\begin{array}{l}\text { löschen } \\
\text { IIsch / ' ’ IS/ }\end{array}$ \\
\hline 29. & $\begin{array}{l}\text { 1.P.Sg.: IM als eh /e }: /+\mathrm{e} / \mathrm{I} / \\
\text { 2.P.Sg.: } \mathrm{IM} \text { als } \mathrm{im} / \mathrm{I} /+\mathrm{st} / \mathrm{st} / \\
\text { 3.P.Sg.: } \mathrm{IM} \text { als } \mathrm{im} / \mathrm{I} /+\mathrm{t} / \mathrm{t} /\end{array}$ & 1 & $\begin{array}{l}\text { nehmen } \\
\mathrm{nIMm} / \mathrm{n} \text { ' Imm/ }\end{array}$ \\
\hline 30. & $\begin{array}{l}\text { 1.P.Sg.: Ä als a } / \mathrm{a}: /+\mathrm{S} \text { als s } / \mathrm{z} /+\mathrm{e} / ə / \\
\text { 2.P.Sg.: } \mathrm{Ä} \text { als ä } / \varepsilon: /+\mathrm{S} \text { als s } / \mathrm{s} /+\mathrm{t} / \mathrm{t} / \\
\text { 3.P.Sg.: } \mathrm{Ä} \text { als } \mathrm{a} / \varepsilon: /+\mathrm{S} \text { als } \mathrm{s} / \mathrm{s} /+\mathrm{t} / \mathrm{t} /\end{array}$ & 1 & $\begin{array}{l}\text { blasen } \\
\text { blÄS /bl ’ y / }\end{array}$ \\
\hline 31. & $\begin{array}{l}\text { 1.P.Sg.: } \mathrm{A} \text { als a } / \mathrm{a}: /+\mathrm{P} \text { als b } / \mathrm{b} /+\mathrm{e} / \mathrm{\partial} / \\
\text { 2.P.Sg.: Ä als ä } / \varepsilon: /+\mathrm{P} \text { als b } / \mathrm{p} /+\mathrm{st} / \mathrm{st} / \\
\text { 3.P.Sg.: Ä als ä } / \varepsilon: /+\mathrm{P} \text { als b } / \mathrm{p} /+\mathrm{t} / \mathrm{t} /\end{array}$ & 1 & 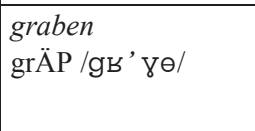 \\
\hline
\end{tabular}




\begin{tabular}{|c|c|c|c|}
\hline & Syntaktische Singularexponenten & $\begin{array}{l}\text { Zahl der Verben, die die } \\
\text { syntaktischen Singula- } \\
\text { rexponenten aufweisen }\end{array}$ & $\begin{array}{c}\text { Beispiel mit } \\
\text { Singularstamm }\end{array}$ \\
\hline 32. & $\begin{array}{l}\text { 1.P.Sg.: } \mathrm{Ä} \text { als a } / \mathrm{a} /+\mathrm{e} / ə / \\
\text { 2.P.Sg.: Ä als ä } / \varepsilon /+\mathrm{st} / \mathrm{st} / \\
\text { 3.P.Sg.: Ä als ä } / \varepsilon /\end{array}$ & 1 & $\begin{array}{l}\text { halten } \\
\text { hÄlt /h’ylt/ }\end{array}$ \\
\hline 33. & $\begin{array}{l}\text { 1.P.Sg.: } \ddot{A} \text { als a } / \mathrm{a}: /+\mathrm{T} \text { als } \mathrm{d} / \mathrm{d} /+\mathrm{e} / ə / \\
\text { 2.P.Sg.: } \mathrm{A} \text { als } \mathrm{ä} / \varepsilon: /+\mathrm{T} \text { als } \mathrm{d} / \mathrm{t} /+\mathrm{st} / \mathrm{st} / \\
\text { 3.P.Sg.: } \mathrm{A} \text { als ä } / \varepsilon: /+\mathrm{T} \text { als dt } / \mathrm{t} /\end{array}$ & 1 & $\begin{array}{l}\text { laden } \\
\text { läT / } 1 \text { ’ } \theta /\end{array}$ \\
\hline 34. & $\begin{array}{l}\text { 1.P.Sg.: } \ddot{A} \text { als a } / \mathrm{a}: /+\mathrm{e} / ə / \\
\text { 2.P.Sg.: } \ddot{\mathrm{A}} \text { als ä } / \varepsilon: /+\mathrm{st} / \mathrm{st} / \\
\text { 3.P.Sg.: } \mathrm{Ä} \text { als ä } / \varepsilon: /+\mathrm{t} / \mathrm{t} /\end{array}$ & 1 & $\begin{array}{l}\text { schlafen } \\
\text { schlÄf / I ' } \mathrm{\gamma f} /\end{array}$ \\
\hline 35. & 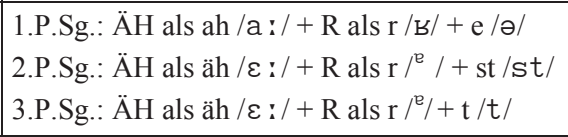 & 1 & $\begin{array}{l}\text { fahren } \\
\text { fÄHR / f' } \gamma^{\mathrm{h}} \mathrm{\Gamma} /\end{array}$ \\
\hline 36. & $\begin{array}{l}\text { 1.P.Sg.: Ö als o } / 0: /+\mathrm{e} / ə / \\
\text { 2.P.Sg.: Ö als ö } / \varnothing: /+\mathrm{t} / \mathrm{t} / \\
\text { 3.P.Sg.: Ö als } \mathrm{o} / \varnothing: /+\mathrm{t} / \mathrm{t} /\end{array}$ & 1 & $\begin{array}{l}\text { stoßen } \\
\text { stÖß/St' -s/ }\end{array}$ \\
\hline 37. & $\begin{array}{l}\text { 1.P.Sg.: A als a } / \mathrm{a}: /+\mathrm{be} / \mathrm{bə} / \\
\text { 2.P.Sg.: A als a } / \mathrm{a} / \mathrm{t} \mathrm{st} / \mathrm{st} / \\
\text { 3.P.Sg.: A als a } / \mathrm{a} / \mathrm{t} / \mathrm{t} /\end{array}$ & 1 & $\begin{array}{l}\text { haben } \\
\text { hA /h' a/ }\end{array}$ \\
\hline 38. & $\begin{array}{l}\text { 1.P.Sg.: e /ə/ } \\
\text { 2.P.Sg.: est /əst/ } \\
\text { 3.P.Sg.: t/t/ } \\
\end{array}$ & 1 & $\begin{array}{l}\text { salzen } \\
\mathrm{salz} / \mathrm{z} \text { ' alt.s/ }\end{array}$ \\
\hline 39. & $\begin{array}{l}\text { 1.P.Sg.: } \varnothing \\
\text { 2.P.Sg.: } \mathrm{t} / \mathrm{t} / \\
\text { 3.P.Sg.: } \varnothing \\
\end{array}$ & 1 & 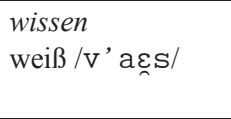 \\
\hline 40. & $\begin{array}{l}\text { 1.P.Sg.: bin /bIn/ } \\
\text { 2.P.Sg.: bist /bIst/ } \\
\text { 3.P.Sg.: ist/ISt/ }\end{array}$ & 1 & $\begin{array}{l}\operatorname{sein} \\
\operatorname{bin} / b^{\prime} \text { In/ } \\
\text { bist /b' Ist/ } \\
\text { ist / ?' Ist/ }\end{array}$ \\
\hline 41. & $\begin{array}{l}\text { 3.P.Sg.: } \\
\text { dünkt/dypkt/ } \\
\text { deucht /dogç t/ }\end{array}$ & 1 & $\begin{array}{l}\text { dünken } \\
\text { dünkt/d' y ykt/ } \\
\text { deucht /d' oæçt/ }\end{array}$ \\
\hline 42. & 3.P.Sg.: geschieht /gəSi:t/ & & $\begin{array}{l}\text { geschehen } \\
\text { geschieht } \\
\text { /gəS'i:t/ }\end{array}$ \\
\hline
\end{tabular}

Die Zahl der syntaktischen Pluralexponenten ist viel geringer: Die durchgeführten Analysen haben 12 Gruppen ergeben (Tabelle Nr. 2), wobei 2 Verben (dünken, geschehen) im Plural nicht vorkommen, sodass die syntaktischen Pluralexponenten sowie Pluralstämme dieser Verben nicht existieren. 
Tabelle Nr. 2: Syntaktische Pluralexponenten für das Präsens Indikativ

\begin{tabular}{|c|c|c|c|}
\hline & Syntaktische Pluralexponenten & $\begin{array}{l}\text { Zahl der Verben, die die } \\
\text { syntaktischen Singula- } \\
\text { rexponenten aufweisen }\end{array}$ & $\begin{array}{l}\text { Beispiel mit } \\
\text { Pluralstamm }\end{array}$ \\
\hline 1. & $\begin{array}{l}\text { 1.P.Pl.: en } / \mathrm{n} / \\
\text { 2.P.P1.: } \mathrm{t} / \mathrm{t} / \\
\text { 3.P.P1.: en } / \mathrm{n} /\end{array}$ & 54 & $\begin{array}{l}\text { befleißen } \\
\text { befleiß/bəfl' aع्s/ }\end{array}$ \\
\hline 2. & $\begin{array}{l}\text { 1.P.Pl.: en /ən/ } \\
\text { 2.P.P1.: t / } / \text { / } \\
\text { 3.P.Pl.: en /ən/ }\end{array}$ & 42 & $\begin{array}{l}\text { beginnen } \\
\text { beginn /bəg' In/ }\end{array}$ \\
\hline 3. & $\begin{array}{l}\text { 1.P.Pl.: } \mathrm{n} / \mathrm{n} / \\
\text { 2.P.Pl.: } \mathrm{t} / \partial \mathrm{t} / \\
\text { 3.P.Pl.: } \mathrm{n} / \mathrm{n} /\end{array}$ & 30 & $\begin{array}{l}\text { bersten } \\
\text { berste } / b^{\prime} \varepsilon^{B} \text { st } /^{7}\end{array}$ \\
\hline 4. & $\begin{array}{l}\text { 1.P.Pl.: } \mathrm{G} \text { als } \mathrm{g} / \mathrm{g} /+\mathrm{en} / \mathrm{y} / \\
\text { 2.P.Pl.: } \mathrm{G} \text { als } \mathrm{g} / \mathrm{k} /+\mathrm{t} / \mathrm{t} / \\
\text { 3.P.Pl.: } \mathrm{G} \text { als } \mathrm{g} / \mathrm{g} /+\mathrm{en} / \mathrm{y} /\end{array}$ & 15 & $\begin{array}{l}\text { bergen } \\
\operatorname{berG} / b^{\prime} \varepsilon^{\mathrm{B}} \mathrm{G} /\end{array}$ \\
\hline 5. & $\begin{array}{l}\text { 1.P.Pl.: B als b } / \mathrm{b} /+ \text { en } / \mathrm{m} / \\
\text { 2.P.Pl.: B als b } / \mathrm{p} /+\mathrm{t} / \mathrm{t} / \\
\text { 3.P.P1.: B als } \mathrm{b} / \mathrm{b} /+\mathrm{en} / \mathrm{m} /\end{array}$ & 15 & $\begin{array}{l}\text { bleiben } \\
\text { bleiB /bl ' a } \_/ /\end{array}$ \\
\hline 6. & $\begin{array}{l}\text { 1.P.Pl.: en } / \mathrm{n} / \\
\text { 2.P.Pl.: } \mathrm{t} / \mathrm{t} / \\
\text { 3.P.P1.: en } / \mathrm{n} /\end{array}$ & 10 & $\begin{array}{l}\text { empfehlen } \\
\text { empfehl / } 2 \varepsilon m p f ' e: 1 /\end{array}$ \\
\hline 7. & $\begin{array}{l}\text { 1.P.Pl.: } \mathrm{R} \text { als } \mathrm{r} / \mathrm{b} /+\mathrm{en} / \partial \mathrm{n} / \\
\text { 2.P.Pl.: } \mathrm{R} \text { als } \mathrm{r} /{ }^{\mathrm{e}} /+\mathrm{t} / \mathrm{t} / \\
\text { 3.P.Pl.: } \mathrm{R} \text { als } \mathrm{r} / \mathrm{b} /+\mathrm{en} / ə \mathrm{n} /\end{array}$ & 8 & 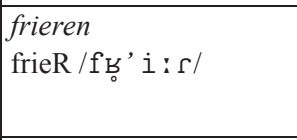 \\
\hline 8. & $\begin{array}{l}\text { 1.P.Pl.: en } / \mathrm{y} / \\
\text { 2.P.Pl.: } \mathrm{t} / \mathrm{t} / \\
\text { 3.P.P1.: en } / \mathrm{y} /\end{array}$ & 7 & $\begin{array}{l}\text { backen } \\
\text { back /b’ak/ }\end{array}$ \\
\hline 9. & $\begin{array}{l}\text { 1.P.P1.: } \mathrm{Z} \text { als s } / \mathrm{z} /+\mathrm{en} / \mathrm{n} / \\
\text { 2.P.Pl.: } \mathrm{Z} \text { als s } / \mathrm{s} /+\mathrm{t} / \mathrm{t} / \\
\text { 3.P.P1.: } \mathrm{Z} \text { als s } / \mathrm{z} /+\mathrm{n} / \mathrm{n} /\end{array}$ & 6 & $\begin{array}{l}\text { blasen } \\
\text { blaZ /bl 'a : } 3 /\end{array}$ \\
\hline 10. & $\begin{array}{l}\text { 1.P.Pl.: } \mathrm{R} \text { als } \mathrm{r} / \mathrm{B} /+\mathrm{en} / \partial \mathrm{n} / \\
\text { 2.P.Pl.: } \mathrm{R} \text { als } \mathrm{r} / \mathrm{s} /+\mathrm{t} / \mathrm{t} / \\
\text { 3.P.Pl.: } \mathrm{R} \text { als } \mathrm{r} / \mathrm{B} /+\mathrm{en} / \partial \mathrm{n} /\end{array}$ & 1 & $\begin{array}{l}\text { fahren } \\
\text { fahR /f' a: } \mathrm{s} /\end{array}$ \\
\hline 11. & $\begin{array}{l}\text { 1.P.Pl.:n /n/ } \\
\text { 2.P.Pl.: } \mathrm{t} / \mathrm{t} / \\
\text { 3.P.Pl.: } \mathrm{n} / \mathrm{n} /\end{array}$ & 1 & $\begin{array}{l}\text { tun } \\
\text { tu /t'u:/ }\end{array}$ \\
\hline 12. & $\begin{array}{l}\text { 1.P.Pl.: sind / } z^{\prime} \text { Int/ } \\
\text { 2.P.Pl.: seid / } z^{\prime} \text { a t. } t / \\
\text { 3.P.Pl.: sind / } z^{\prime} \text { Int/ }\end{array}$ & 1 & \begin{tabular}{|l|}
$\operatorname{sein}$ \\
$\operatorname{sind} / \mathrm{z}^{\prime}$ Int/ \\
$\operatorname{seid} / \mathrm{z}^{\prime}$ ą્t/ \\
$\operatorname{sind} / \mathrm{z}^{\prime}$ Int/
\end{tabular} \\
\hline
\end{tabular}

\footnotetext{
${ }^{7}$ In dieser Gruppe entspricht der orthographische Pluralstamm dem orthoepischen Pluralstamm nicht.
} 
Aufgrund der Zuordnung aller Verben den ermittelten Gruppen der syntaktischen Singular- und Pluralexponenten ergeben sich ihre Konjugationsmuster. Demzufolge hat z.B. das Verb beginnen (der Singular- und Pluralstamm: beginn /bəg' In/) folgendes Konjugationsmuster:

\begin{tabular}{|c|c|}
\hline Singular & Plural \\
\hline 1. Person e /ə/ & 1. Person en /ən/ \\
\hline 2. Person st /st/ & 2. Person t / t/ \\
\hline 3. Person $t / t /$ & 3. Person en /ən / \\
\hline
\end{tabular}

Nach diesem Konjugationsmuster werden auch folgende Verben gebeugt: brennen, bringen, dingen, dringen, fliehen, gedeihen, gehen, gelingen, gewinnen, glimmen, hängen, hauen, kennen, klimmen, kommen, leihen, misslingen, nennen, rennen, ringen, rinnen, schlingen, schreien, schwimmen, schwingen, singen, sinnen, speien, spinnen, springen, stehen, wringen, zeihen, ziehen, zwingen. Die Singular- und Pluralstämme der Verben, die nach diesem Muster konjugiert werden, sind identisch und gehen aus auf:

- Nasale: /m/, /n/ und /y/ (beginnen, brennen, bringen, dingen, dringen, gelingen, gewinnen, glimmen, hängen, kennen, klimmen, kommen, misslingen, nennen, rennen, ringen, rinnen, schlingen, schwimmen, schwingen, singen, sinnen, spinnen, springen, wringen, zwingen),

- Vollvokale: /i :/(fliehen, ziehen),/e : (gehen, stehen),

- Halbvokale: /E्/ (gedeihen, leihen, schreien, speien, zeihen), /o/ (hauen).

Die vergleichende Analyse der Singular- und Pluralstämme ergibt die sog. neutralen Pluralstämme, die die Ermittlung der Singular- und Pluralmarker (d.h. der Elemente, die den Singular bzw. Plural anzeigen) ermöglichen. Zu bemerken ist aber, dass syntaktische Exponenten bestimmter Verben gleichzeitig als ihre Zahlmarker fungieren, d.h. sie sind bifunktional.

Die Analyse hat 40 Typen von Singularmarkern ergeben. Die syntaktischen Singularexponenten der Gruppen Nr. 1, 3-5, 7, 12, 38 und 40-42 (siehe: Tabelle Nr. 1) sind gleichzeitig ihre Singularmarker. Im Falle der Gruppe Nr. 2 haben wir mit bifunktionalen syntaktischen Singularexponenten nur auf der orthographischen Ebene zu tun. Auf der phonologischen Ebene sind die syntaktischen Singularexponenten monofunktional ${ }^{9}$. Die folgende Tabelle präsentiert die Singularmarker der weiteren unregelmäßigen Verben, geordnet nach der Häufigkeit ihres Auftretens.

\footnotetext{
${ }^{8}$ Vgl. auch dazu: Darski 1999: 100.

${ }^{9}$ Vgl. dazu: die Gruppe Nr. 2 von syntaktischen Singularexponenten in der Tabelle Nr. 1 und den Typ Nr. 2 von Singularmarkern in der Tabelle Nr. 3.
} 
Tabelle Nr. 3: Syntaktische Singularmarker der unregelmäßigen Verben

\begin{tabular}{|c|c|c|c|}
\hline & Typ des Singularmarkes & $\begin{array}{c}\text { Zahl der Verben, die diesen } \\
\text { Typ des Singularmarkers } \\
\text { aufweisen }\end{array}$ & $\begin{array}{l}\text { Beispiel mit neutralem } \\
\text { Flexionsstamm }\end{array}$ \\
\hline 1. & $\begin{array}{l}\text { E als i /I/ } \\
\mathrm{e} / ə /\end{array}$ & 22 & $\begin{array}{l}\text { bersten } \\
\text { bErst /b’ } \varepsilon^{\mathrm{B}} \mathrm{st} /\end{array}$ \\
\hline 2. & $\begin{array}{l}\emptyset / ə / \\
\text { st /əst/ } \\
\mathrm{t} / \text { /ət/ }\end{array}$ & 19 & $\begin{array}{l}\text { bieten } \\
\text { biete /b’ i : t/ }\end{array}$ \\
\hline 3. & $\begin{array}{l}\text { A als ä } / \varepsilon / \\
\mathrm{e} / ə /\end{array}$ & 7 & $\begin{array}{l}\text { backen } \\
\text { bAck /b'ak/ }\end{array}$ \\
\hline 4. & $\begin{array}{l}\text { EH als ieh /i :/ } \\
\text { e /ə/ }\end{array}$ & 4 & $\begin{array}{l}\text { befehlen } \\
\text { befEHl /bəf' } \varepsilon^{\mathrm{h}} \mathrm{l} /\end{array}$ \\
\hline 5. & 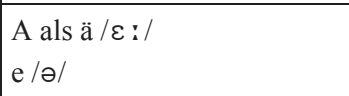 & 3 & $\begin{array}{l}\text { braten } \\
\text { brAt/br'at/ }\end{array}$ \\
\hline 6. & $\begin{array}{l}\mathrm{E} \text { als } \mathrm{i} / \mathrm{I} / \\
\mathrm{B} \text { als } \mathrm{b} / \mathrm{b} /+\mathrm{e} / \mathrm{\partial} /\end{array}$ & 3 & $\begin{array}{l}\text { sterben } \\
\operatorname{stErB} / \int t^{\prime} \varepsilon^{\mathrm{B}} \beta /\end{array}$ \\
\hline 7. & $\begin{array}{l}\mathrm{E} \text { als i /I/ } \\
\mathrm{t} / \mathrm{t} /+\mathrm{e} / \mathrm{\theta} /\end{array}$ & 2 & $\begin{array}{l}\text { fechten } \\
\text { fEch /f' } \varepsilon \mathrm{c} /\end{array}$ \\
\hline 8. & $\begin{array}{l}\text { E als i / I/ } \\
\text { e /ə/ } \\
\text { est /əst/ }\end{array}$ & 2 & $\begin{array}{l}\text { fechten } \\
\text { fEcht /f' } \varepsilon c ̧ t /\end{array}$ \\
\hline 9. & $\begin{array}{l}\text { AU als äu /ə@// } \\
\text { e /ə/ }\end{array}$ & 2 & $\begin{array}{l}\text { laufen } \\
\text { 1AUf / ' avf/ }\end{array}$ \\
\hline 10. & 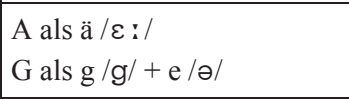 & 2 & $\begin{array}{l}\text { schlagen } \\
\text { schlAG / I I'ag/ }\end{array}$ \\
\hline 11. & st/st/ & 1 & $\begin{array}{l}\text { sollen } \\
\text { soll /z' ol/ }\end{array}$ \\
\hline 12. & $\begin{array}{l}\mathrm{E} \text { als i /I/ } \\
\mathrm{G} \text { als g/g/ + e /ə/ }\end{array}$ & 1 & $\begin{array}{l}\text { bergen } \\
\mathrm{bErG} / \mathrm{b} \mathcal{E}^{\mathrm{B}} \mathrm{G} /\end{array}$ \\
\hline 13. & $\begin{array}{l}\mathrm{E} \text { als } \mathrm{i} / \mathrm{i}: / \\
\mathrm{B} \text { als } \mathrm{b} / \mathrm{b} /+\mathrm{e} / \mathrm{a} /\end{array}$ & 1 & $\begin{array}{l}\text { geben } \\
\mathrm{gEB} / \mathrm{g} \text { ' } \varepsilon \beta /\end{array}$ \\
\hline 14. & $\begin{array}{l}\text { E als ie } / \mathrm{i}: / \\
\mathrm{Z} \text { als s/z/+e/ə/ }\end{array}$ & 1 & $\begin{array}{l}\text { lesen } \\
1 \mathrm{EZ} / 1 \text { ' } \varepsilon 3 /\end{array}$ \\
\hline 15. & $\begin{array}{l}\text { I als i /I/ } \\
\text { de /də/ }\end{array}$ & 1 & $\begin{array}{l}\text { werden } \\
\mathrm{wEr} / \mathrm{v} \varepsilon^{\mathrm{B}} /\end{array}$ \\
\hline 16. & $\begin{array}{l}\text { EH als im /I/ } \\
\text { e /ə/ }\end{array}$ & 1 & $\begin{array}{l}\text { nehmen } \\
\mathrm{nEHm} / \mathrm{n}{ }^{\prime} \varepsilon^{\mathrm{h}} \mathrm{m} /\end{array}$ \\
\hline 17. & $\begin{array}{l}\mathrm{A} \text { als ä/E:/ } \\
\mathrm{Z} \text { als s } / \mathbf{Z} /+\mathrm{e} / \oslash /\end{array}$ & 1 & $\begin{array}{l}\text { blasen } \\
\text { blAZ /bl'az/ }\end{array}$ \\
\hline
\end{tabular}




\begin{tabular}{|c|c|c|c|}
\hline & Typ des Singularmarkes & $\begin{array}{l}\text { Zahl der Verben, die diesen } \\
\text { Typ des Singularmarkers } \\
\text { aufweisen }\end{array}$ & $\begin{array}{l}\text { Beispiel mit neutralem } \\
\text { Flexionsstamm }\end{array}$ \\
\hline 18. & A als a /a/ & 1 & $\begin{array}{l}\text { dürfen } \\
\text { dArf } / \mathrm{d}^{\prime} \mathrm{a}^{\mathrm{B}} \mathrm{f} /\end{array}$ \\
\hline 19. & 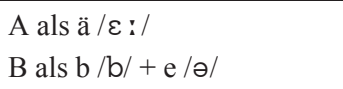 & 1 & $\begin{array}{l}\text { graben } \\
\text { grAB /gb' a } \beta /\end{array}$ \\
\hline 20. & $\begin{array}{l}\text { A als a /a/ } \\
\text { be /bə/ }\end{array}$ & 1 & $\begin{array}{l}\text { haben } \\
\text { hA/h' } \mathrm{a} /\end{array}$ \\
\hline 21. & 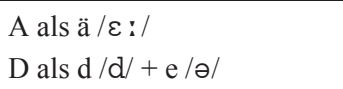 & 1 & $\begin{array}{l}\text { laden } \\
1 \mathrm{AD} / 1 \text { ' að/ }\end{array}$ \\
\hline 22. & A als a /a:/ & 1 & $\begin{array}{l}\text { mögen } \\
\mathrm{mAG} / \mathrm{m} \text { ' aG/ }\end{array}$ \\
\hline 23. & $\begin{array}{l}\text { AH als äh } / \varepsilon: / \\
\mathrm{e} / ə /\end{array}$ & 1 & $\begin{array}{l}\text { fahren } \\
\text { fAHR / } \text { ' }^{\text {h }}{ }^{\mathrm{h}} \mathrm{\rho} /\end{array}$ \\
\hline 24. & $\begin{array}{l}\ddot{\mathrm{A}} \text { als ie } / \mathrm{i}: / \\
\mathrm{R} \text { als } \mathrm{r} / \mathrm{b} /+\mathrm{e} / ə /\end{array}$ & 1 & $\begin{array}{l}\text { gebären } \\
\text { gebÄR /gəb' ' } г /\end{array}$ \\
\hline 25. & $\begin{array}{l}\text { I als ei /ą̧/ } \\
\mathrm{S} \text { als } \beta / \mathrm{s} /\end{array}$ & 1 & $\begin{array}{l}\text { wissen } \\
\text { wIS /V' I S/ }\end{array}$ \\
\hline 26. & $\begin{array}{l}\mathrm{O} \text { als ö /ø:/ } \\
\mathrm{e} / \partial /\end{array}$ & 1 & $\begin{array}{l}\text { stoßen } \\
\text { stOß/St’ Øs/ }\end{array}$ \\
\hline 27. & $\mathrm{O}$ als i /I/ & 1 & \begin{tabular}{|l|} 
wollen \\
wOll /v'øl/
\end{tabular} \\
\hline 28. & Ö als a /a/ & 1 & $\begin{array}{l}\text { können } \\
\text { kÖnn /k' -n/ }\end{array}$ \\
\hline 29. & $\begin{array}{l}\text { Ö als i /I/ } \\
\mathrm{e} / \partial /\end{array}$ & 1 & $\begin{array}{l}\text { löschen } \\
\text { 1Ösch /1' - S/ }\end{array}$ \\
\hline 30. & $\mathrm{U}$ als u $/ \mathrm{v} /$ & 1 & $\begin{array}{l}\text { müssen } \\
\mathrm{mUs} / \mathrm{m} \text { ' Us/ }\end{array}$ \\
\hline
\end{tabular}

Im Falle von Pluralmarkern der unregelmäßigen Verben werden 21 Typen ermittelt, wobei zwei Verben (dünken, geschehen) im Plural nicht vorkommen, sodass die Pluralmarker dieser Verben auch nicht existieren.

Die syntaktischen Pluralexponenten der Gruppen Nr. 7-9, 11 und 12 (siehe: Tabelle Nr. 2) sind gleichzeitig ihre Pluralmarker.

Bifunktional sind auch die syntaktischen Pluralexponenten der meisten Verben der Gruppen Nr. 1-6. Der Gruppe Nr. 1 entfallen jedoch die Verben dürfen, fechten, flechten, müssen und wissen; der Gruppe 2 entfällt das Verb können; der Gruppe 3 werden; der Gruppe 4 - mögen; der Gruppe 5 - haben und der Gruppe 6 -wollen (siehe: Tabelle Nr. 2). Hinzu kommt noch das Verb fahren (siehe: Tabelle Nr. 2, die 
Gruppe Nr. 10), dessen Pluralmarker sich mit syntaktischen Pluralexponenten nicht decken, d.h. syntaktische Exponenten sind monofunktional. Die Pluralmarker der oben aufgezählten Verben illustriert die Tabelle Nr. 4.

Tabelle Nr. 4: Syntaktische Pluralmarker der unregelmäßigen Verben

\begin{tabular}{|c|c|c|}
\hline & $\begin{array}{l}\text { Das Verb mit seinem neutralen } \\
\text { Flexionsstamm }\end{array}$ & Pluralmarker \\
\hline 1. & $\begin{array}{l}\text { dürfen } \\
\text { dArf /d' } a^{\mathrm{B}} \mathrm{f} /\end{array}$ & $\mathrm{A}$ als $\ddot{\mathrm{u}} / \mathrm{Y} /$ \\
\hline 2. & $\begin{array}{l}\text { fahren } \\
\text { fAHR } / \mathrm{f}^{\prime} \mathrm{a}^{\mathrm{h}} \mathrm{\rho} /\end{array}$ & $\begin{array}{l}\text { en /ən/ } \\
\mathrm{R} \text { als r/B/ } \\
\text { en /ən/ }\end{array}$ \\
\hline 3. & 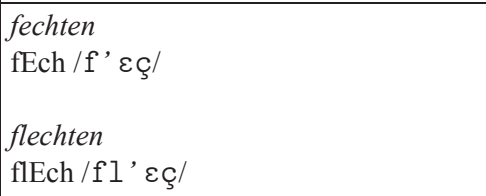 & $\begin{array}{l}\text { ten / } \operatorname{tn}_{1} / \\
\text { tet / tət/ } \\
\text { ten / } \operatorname{tn}_{1} /\end{array}$ \\
\hline 4. & $\begin{array}{l}\text { haben } \\
\text { hA /h' a/ }\end{array}$ & $\begin{array}{l}\text { ben } / \mathrm{bm} / \\
\text { bt } / \mathrm{pt} / \\
\text { ben } / \mathrm{bm} /\end{array}$ \\
\hline 5. & $\begin{array}{l}\text { können } \\
\mathrm{kÖnn} / \mathrm{k}{ }^{\prime}-\mathrm{n} /\end{array}$ & Ö als ö /œ/ \\
\hline 6. & $\begin{array}{l}\text { mögen } \\
\mathrm{mAG} / \mathrm{m} \text { ’aG/ }\end{array}$ & $\begin{array}{l}\text { A als ö/ø:/ } \\
\text { G als g /g/ }\end{array}$ \\
\hline 7. & \begin{tabular}{|l|} 
müssen \\
$\mathrm{mUs} / \mathrm{m}$ ' Us/
\end{tabular} & $\mathrm{U}$ als ü $/ \mathrm{Y} /$ \\
\hline 8. & $\begin{array}{l}\text { werden } \\
\mathrm{wEr} / \mathrm{v} \varepsilon^{\mathrm{B} /}\end{array}$ & $\begin{array}{l}\operatorname{den} / \mathrm{dn}_{1} / \\
\operatorname{det} / \mathrm{d} \partial \mathrm{t} / \\
\operatorname{den} / \mathrm{dn}_{1} /\end{array}$ \\
\hline 9. & $\begin{array}{l}\text { wissen } \\
\text { wIS /V' I S/ }\end{array}$ & $\begin{array}{l}\text { I als i /I/ } \\
\mathrm{S} \text { als ss /S/ }\end{array}$ \\
\hline 10. & \begin{tabular}{|l|} 
wollen \\
wOll /v' øl/
\end{tabular} & $\mathrm{O}$ als o $/ \mathrm{O} /$ \\
\hline
\end{tabular}

Die durchgeführten Analysen beschränkten sich auf syntaktische Exponenten und Zahlmarker der unregelmäßigen Verben im Präsens Indikativ. Die hier präsentierten Ergebnisse zeugen jedoch eindeutig davon, dass die Ermittlung syntaktischer Exponenten und Zahlmarker weiterer Verben und in anderen Tempusformen und Modi wissenschaftlich exakt und widerspruchsfrei erfolgen kann, was auch im didaktischen Bereich Anwendung finden sollte. 


\section{Literatur}

Darski, J. (1999): Bildung der Verbformen im Standarddeutschen. Tübingen: Stauffenburg.

Darski, J. (2004): Linguistisches Analysemodell. Definitionen grundlegender grammatischer Begriffe. 2., völlig neu bearbeitete und ergänzte Auflage. Poznań: Wydawnictwo Naukowe UAM.

Darski, J. (2010): Deutsche Grammatik. Ein völlig neuer Ansatz. Frankfurt am Main et al.: Peter Lang.

DUDEN. Die Grammatik. (2005) 7., völlig neu erarbeitete und erweiterte Auflage. Herausgeben von der Dudenredaktion. Bearbeitet von P. Eisenberg, J. Peters, P. Gallmann, C. Fabricius-Hansen, D. Nübling, I. Barz, T.A. Fritz, R. Fiehler. Mannheim, Leipzig, Wien, Zürich: Dudenverlag.

Eisenberg, P. (1989): Grundriß der deutschen Grammatik. 2., überarbeitete und erweiterte Auflage. Stuttgart: J:B. Metzlersche Verlagsbuchhandlung.

Engel, U. (1977): Syntax der deutschen Gegenwartssprache. Berlin: Erich Schmidt.

Engel, U. (2004): Deutsche Grammatik, Neubearbeitung. München: Iudicum.

Helbig, G., Buscha, J. (2001): Deutsche Grammatik. Ein Handbuch für Ausländerunterricht. LeipzigBerlin-München-Wien-Zürich-New York: Enzyklopädie Langenscheidt.

Jung, W. (1955): Kleine Grammatik der deutschen Sprache. Satz- und Beziehungslehre. Leipzig: VEB Bibliographisches Institut.

Krech, E.-M., Stock, E., Hirschfeld, U., Anders, L.Ch. (Hrsg.) (2009): Deutsches Aussprachewörterbuch. Berlin-New York: Walter de Gruyter.

Weinrich, H. (1993): Textgrammatik der deutschen Sprache. Mannheim, Leipzig, Wien, Zürich: Dudenverlag. 